\title{
Endogenous platforms: the case of many parties
}

\author{
Andrei M. Gomberg • Francisco Marhuenda • \\ Ignacio Ortuño-Ortín
}

Revised: 15 June 2006 / Published online: 1 November 2006

(C) Springer-Verlag 2006

\begin{abstract}
We analyze existence of equilibrium in a one-dimensional model of endogenous party platforms and more than two parties. The platforms proposed by parties depend on their membership composition. The policy implemented is a function of the different proposals and the vote distribution among such proposals. It is shown that if voters are sincere there is always an equilibrium regardless of the number of parties. In the case of strategic voting behavior, existence of equilibrium can be shown provided a subadditivity condition on the outcome function holds.
\end{abstract}

\section{Introduction}

In recent years there have been a number of papers in political economy dealing with the issue of endogenous platform formation. The main idea of this literature consists in recognizing that to understand the way parties choose their platforms one should analyze both the "external" politics of the parties as well as the "internal" politics. The "external politics" refers to the connection

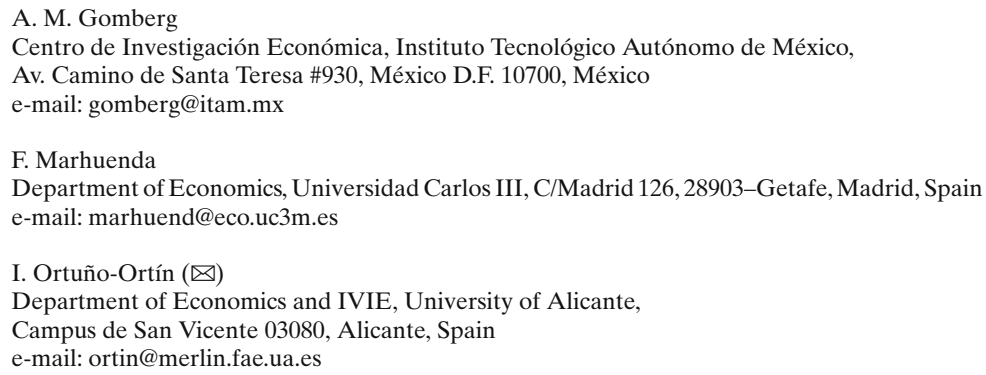


between the political platform adopted by a party and the response of the voters to it. The "internal politics" refers to the connection between the party's membership and its platform. These two sides of the party's political problem can be solved simultaneously, for instance, by assuming that, at equilibrium, the proposal put forward by the party, which depends on its membership, should be preferred by all its members to any of the policies proposed by the other parties. An early paper using this idea in a political competition framework to define an equilibrium concept that renders the party ideology endogenous was Baron (1993). This equilibrium concept is closely related to the one used in the "voting with one's feet" models originally developed in the context of local public goods (see Caplin and Nalebuff 1997 for an abstract framework that encompasses both the party formation and local public finance applications). Ortuño-Ortín and Roemer (1998) use the same equilibrium concept in an example in the one-dimensional policy space. Roemer (1999) deals with a two-dimensional policy space problem but the nature of the "external" policy equilibrium differs from those standard in the literature. In a closely related line of research, papers such as Aldrich (1983), Gerber and Ortuño-Ortín (1998), and Poutvaara (2003) have considered the interrelationship between partisan policy platforms and political activism.

Gomberg, Marhuenda and Ortuño-Ortín (2004) (henceforth, GMO) consider a general model of two parties competing in a multidimensional policy space. Party platforms depend on the composition of the parties' membership (interpreted as its internal primary electorate), whereas party membership depends on the proposed party platforms. Individuals vote strategically and the overall social outcome is taken to be a weighted average of party platforms and overall equilibrium is obtained when no group of voters can shift the social outcome in its favor by switching parties (external equilibrium) and the party platforms are consistent with their electorate (internal equilibrium). They show that existence of overall equilibrium with distinct platforms ("pluralistic equilibrium") in the two-party case depends on the odd-even dimensionality of the policy space. This result follows from the theoretical development of Caplin and Nalebuff (1997) and Gomberg (2004), which showed that conditions necessary to ensure existence of pluralistic equilibria in twojurisdiction (including two-party) models are sensitive to the parity of policy space dimension. The apparently paradoxical result is somewhat clarified by the example in Gomberg (2005), which suggests a more complex interaction between the number of parties and the dimensionality of the policy space.

This paper takes up the GMO framework and studies the issue of pluralistic equilibrium existence in the case of more than two parties. However, we restrict the analysis to the one-dimensional policy space. In common with the abovecited papers, the number of available "shell" parties is exogenously given. Thus, we do not deal with a model of endogenous number of parties. This assumption renders the approach here different from the one in Levy (2004) and from the popular models of "citizen-candidates" (Osborne and Slivinski 1996 and Besley and Coate 1997) in which candidates are endogenously determined. 
Nonetheless, the number of parties that in equilibrium have non-negligible membership is endogenous in our model. In particular, we are interested in establishing conditions for existence of equilibria in which all available parties receive a strictly positive mass of votes. It turns out that such existence results crucially depend on the specifics of the model of interparty ("external") competition. Namely, the results depend on the nature of the voters' behavior (whether the voting is sincere or strategic) and on the manner in which voting results are aggregated into the final policy choice by the society (i.e., on whether the political system is favoring smaller or larger parties).

In the more straightforward case of sincere voting, where all agents vote for the party with the most appealing platform irrespective of the impact on final policy, we show that overall pluralistic equilibrium exists for any number of parties under fairly standard assumptions. It should be noted that formally this result is closely related to a string of sorting equilibrium existence results in local public goods economies dating back to Westhoff (1977).

If voting behavior is strategic (as in GMO), the results crucially depend on the nature of the electoral system. We study two cases here depending on either the superadditive or subadditive nature of such relation between vote share and the influence on the outcome policy. In the superadditive case, a single party receiving the same vote share as the sum of the vote shares received by two parties obtains a greater weight than the sum of the weights of those two parties. This superadditivity assumption is related to the "economies of party size" analyzed in Osborne and Tourky (2004). In this case, and for the one-dimensional policy space, Gerber and Ortuño-Ortín (1998) show that whatever the profile of party platforms there can be no "external" voting equilibrium with more than two parties receiving a strictly positive vote share. Thus, and following on the suggestion in Osborne and Tourky (2004), we consider the subadditive case (i.e., the case where two separate parties together are more powerful than a single "merged" party). For this case, we provide general conditions for existence of voting equilibrium with three parties receiving a positive vote share. Thus, our results can be viewed as providing one formalization to the so-called Duverger's Hypothesis, that claims that multiparty systems are favored by the proportional representation systems. We proceed to adapt the proof of existence of overall equilibrium in the sincere case to deal with the strategic behavior.

The rest of the paper is organized as follows. Section 2 introduces a general spatial model of multiparty interaction along the lines of GMO. Section 3 presents the proof of equilibrium existence of equilibrium in a voting game with exogenous party platforms. Section 4 defines an overall multiparty pluralistic equilibrium, Sect. 5 provides a proof of its existence in the sincere voting case, while Sect. 6 extends the existence result to the strategic voting case.

\section{The general model}

The model in this section is based on GMO. The society has to implement a vector of policies $x \in Z$, where $Z$ is a non-empty compact and convex subset 
of $\mathbb{R}$. We denote by $A$ the set of types of agents. Each agent $\alpha \in A$ has Euclidean preferences represented by the utility function

$$
u(x, \alpha)=-|x-\alpha|, \quad x \in A
$$

We can, therefore, identify an agent with his ideal vector in $A$. We assume that the set of agents $A$ is a compact and convex non-singleton subset of $Z$ and so, without loss of generality, we will take $A=Z=[0,1]$.

The distribution of agents is described by a nowhere vanishing, continuous density function $f$. We let $F$ denote the associated distribution function. The measure of a Lebesgue measurable subset $B$ of $A$ according to this density is denoted by $\mu(B)$.

There are $m \geq 2$ parties. Individuals are free to join any of the parties, resulting in a population partition $C=\left\{C_{1}, \ldots, C_{m}\right\}$ of $A$, consisting of non-empty, (Lebesgue) measurable subsets. The policy proposed by party $i=1, \ldots, m$ is denoted by $P_{i}(C)$ and is determined as follows

Assumption 2.1 There is a mapping $P$ that assigns to each partition $\left\{C_{1}, \ldots, C_{m}\right\}$ of $A$ the policies $P(C)=\left(P_{1}(C), \ldots, P_{m}(C)\right)$ proposed by the parties. The mapping $P$ satisfies that, $P_{i}(C) \in C_{i}$ for each $i=1, \ldots, m$.

Thus, $P$ is an exogenous mapping that assigns to each subset $B \subset A$, the policy of party $B$. One may interpret $P$ as a profile of party statutes that establish the manner in which preferences of party memberships aggregate into a platform. Typical examples of a $P_{i}$ would be the median-voter and mean-voter rules, which tell each party $i$ to choose the feasible $\left(P_{i}(C) \in C_{i}\right)$ policy that is the best for, respectively, its median or mean member. ${ }^{1}$ Furthermore, we shall assume that the policy chosen by each party enjoys the support of a minimum fraction of the total population in that party. Formally,

Assumption 2.2 There is a $\delta>0$ such that if $C=\left\{C_{1}, \ldots, C_{m}\right\}$ is a partition of $A$ consisting of measurable subsets with $\mu\left(C_{i}\right)>0$, then for all $i=1, \ldots, m$ and $y \in C_{i}$, we have

$$
\frac{\mu\left(\left\{\alpha \in C_{i}: u\left(P_{i}(C), \alpha\right) \geq u(y, \alpha)\right\}\right)}{\mu\left(C_{i}\right)} \geq \delta
$$

This assumption says that the proposal of a party should obtain a minimum support among its members.

Given a set of proposals, $p=\left(p_{1}, \ldots, p_{m}\right)$, individuals shall vote, in a manner explained below, inducing some population partition $C$. The corresponding

1 Notice that if $C_{i}$ is an interval, this amounts to simply choosing the ideal point of, respectively, the median or mean voter. If $C_{i}$ is non-convex, though, the rule definitions may be ambiguous. We shall resolve the ambiguity for the mean-voter rule by always assuming that if there is more than one point in $C_{i}$ at the smallest distance from the mean voter, the leftmost of these is chosen. For the median-voter rule, if there are multiple median points in $C_{i}$ we shall always take the leftmost of these. Given the nature of equilibria we shall construct, these tie-breaking assumptions are without loss of generality. 
vote shares are represented by a vector $w(C) \in \Delta^{m-1}=\left\{\left(x_{1}, \ldots, x_{n}\right) \in\right.$ $\left.\mathbb{R}_{+}^{m}: x_{1}+\cdots+x_{n}=1\right\}$, the $m-1$ dimensional simplex. When the underlying partition is clear we shall just write $w$.

The overall policy outcome depends on the manner in which the vote divides between the parties, as well as on the propositions that are on offer and on the type of political institution in place. We assume that the relevant political institutions are fixed so that the policy outcome can be seen as depending exclusively on the proposals and the vote distribution. Thus, there is some outcome function $T: Z^{m} \times \Delta^{m-1} \rightarrow Z$. While, in principle, a general set of outcome functions may be analyzed, we restrict ourselves to special classes of these. In particular, in this paper we shall focus on the "convex combination" outcome functions (see Alesina and Rosenthal 1997 and Ortuño-Ortín 1997).

Assumption 2.3 $T(p, w)=\sum_{j=1}^{m} g^{j}(w) p^{j}$, where $g \equiv\left(g^{1}, \ldots, g^{m}\right)$ is a continuous function from $\Delta^{m-1}$ to itself.

The idea behind this assumption is that all parties with electoral representation have some influence on the policy implemented. Thus, our model, technically, does not include "winner takes all" models. However, the influence exerted by minority parties could be arbitrary small, meaning that the winnertake all model can be arbitrarily well approximated by a sequence of functions $T$.

The following assumption on the outcome function shall be imposed throughout.

\section{Assumption 2.4}

(1) For every $i=1, \ldots, m$, the weight $g^{i}(w)$ assigned to party $i$ is strictly increasing in the vote share $w^{i}$ obtained by that party.

(2) For every party $i=1, \ldots, m$, we have that $g^{i}(w)=0$ whenever $w^{i}=0$ and $g^{i}(w)=1$ whenever $w^{i}=1$

(3) (anonymity) If $w^{i}=w^{j}$ then $g^{i}(w)=g^{j}(w)$.

In terms of the vote-share impact function $g$, we shall concentrate on two general families in this paper: superadditive and subadditive.

Definition 2.5 The function $g$ is superadditive if for any vector of vote share $w$ with $w^{i} \geq w^{j}$ for some $i \neq j$ and for any $\varepsilon \in\left[0, w^{j}\right]$ we have that $g^{i}(w)+g^{j}(w) \leq$ $g^{i}(\bar{w})+g^{j}(\bar{w})$, where $w^{k}=\bar{w}^{k}$ for all $k \neq i, j$ and $\bar{w}^{i}=w^{i}+\varepsilon, \bar{w}^{j}=w^{j}-\varepsilon$. We say that $g$ is subadditive if the reverse inequality happens, i.e. if $g^{i}(w)+g^{j}(w)>$ $g^{i}(\bar{w})+g^{j}(\bar{w})$.

In words, the superadditivity of $g$ can be interpreted as implying the political system favoring large parties, while the subadditivity of $g$ indicates a bias towards small parties.

Under the assumption of Euclidean preferences, given the policy proposals represented by $p$ and the voting pattern represented by $w$, the payoff enjoyed by an individual of type $\alpha$ is given by $u(T(p, w) ; \alpha)=-|\alpha-T(p, w)|$. Regarding 
the way agents decide their vote, two alternatives are considered in this paper,

(1) Agents vote sincerely.

(2) Agents vote strategically.

When agents behave sincerely, they vote for their most preferred proposal. Assuming the party platforms are distinct, we may reorder parties so that $p_{1}<p_{2}<\cdots<p_{m}$. Thus, taking the convention that $a_{0}=0, a_{m}=1$, the set of voters who favor the proposal of party $i=1, \ldots, m$ is

$$
C_{i}(p)=\left[a_{i-1}, a_{i}\right]
$$

where $a_{i}=\frac{p_{i}+p_{i+1}}{2}$.

In the strategic case, agents care about the outcome $T(p, w)$. Thus, it might happen that some agents do not vote for their most preferred policy. Since any agent in a continuum is negligible, in order for the strategic voting to be non-trivial, as in GMO, we choose an equilibrium concept that involves the possibility of coalitional deviations [in this, we follow such papers as Alesina and Rosenthal (1997) and Gerber and Ortuño-Ortín (1998)]. Thus, given a vector of proposals $p$, the Strong Nash equilibrium (SNE) of Aumann (1959) requires that the associated population partition $C$ is such that no coalition of positive measure of agents wants to deviate and vote in a way different from the one prescribed by the equilibrium strategy. That is, any voting deviation changes the outcome $T(p, w)$ in a direction such that some of the members in the deviation coalition end up worse off.

\section{Existence of equilibrium in the voting game with strategic voters}

In the case of sincere voting behavior, existence of equilibrium in the voting game is automatic. Each agent votes for the closest proposal to his most preferred policy, and the corresponding population partition is given by Eq. 2.1 in the previous section. Notice that, in this case, agents do not care about the outcome $T(p, w(C))$ and, consequently, the voting equilibrium does not depend on our assumption on the type of additivity of the function $g$.

The case of strategic voting behavior is more complicated and, moreover, existence of equilibrium depends on the nature of the function $g$. In a more general model, Gerber and Ortuño-Ortín (1998) show that if $g$ is superadditive $^{2}$ and the policy space is one-dimensional, there is no equilibrium for which more than two parties receive a vote share of (strictly) positive measure. A similar result is provided, in a different but related environment, by Osborne and Tourky (2004). They show that, if there exist economies of party size, there is no equilibrium with more than two parties. The idea behind these results is very intuitive. Consider an equilibrium with three parties, each of them obtaining a positive vote share. Let the policy proposals be $p_{1}<p_{2}<p_{3}$. Say that

\footnotetext{
2 Their definition of superadditivity is weaker than the one adopted in this paper.
} 
the outcome $T(p, w(C))$ is such that $p_{2}<T(p, w(C))<p_{3}$. Superadditivity of $g$ implies that if some (positive mass) of agents voting for $p_{2}$ deviate and vote for $p_{1}$ instead, the outcome would move toward the left. It is not difficult to show that there always exists such a set of agents among the voters of $p_{2}$ whose ideal policies are to the left of $T(p, w(C))$. Those agents improve by deviating from the original strategy and voting for $p_{1}$ instead. Thus, there is no three(or more) party equilibrium when the policy space is one-dimensional and $g$ is superadditive.

This negative result seems to be quite robust and Osborne and Tourky (2004) suggest that existence of equilibrium with more than two parties can only be explained if the dimension of the policy space is higher than one and/or there are diseconomies of party size (in our environment this is an interpretation of subadditivity of $g$ ).

This paper does not explore whether increasing the dimensionality of the policy space solves the equilibrium existence problem or not. ${ }^{3}$ In this section, and following the suggestion in Osborne and Tourky (2004), we study the possible existence of voting equilibrium in the case of subadditivity of $g$ in the onedimensional policy space with more than two parties. For simplicity, we will restrict our analysis to the case of three parties, even though, the same ideas could be applied to any number of parties and similar results could be obtained. Considering the general case, however, would have substantially complicated the analysis below without providing additional intuitions, so we will state the results and provide the proofs only for the three-party case.

Assumption 3.1 There are three parties, agents vote strategically and the function $g$ is subadditive.

Let $p=\left\{p_{1}, p_{2}, p_{3}\right\}$ be the parties's proposals. We analyze the case in which these proposals are distinct and w.l.o.g. we assume that $0 \leq p_{1}<p_{2}<p_{3} \leq 1$. Each agent in $Z$ has to vote for one element of $p$. Thus, the strategy space for each agent $\alpha \in Z$ is $E_{\alpha}=p$. We define $E=\prod_{\alpha \in Z} E_{\alpha}$. Clearly, an element $e \in E$ specifies the way each agent votes. Thus, the vector $p$ and the chosen strategy profile $e$ determine a voting partition $C=\left(C_{1}, C_{2}, C_{3}\right)$ of $Z$. It might be convenient to write such partition as $C(e)$, a function of the strategies chosen by the agents. The outcome function is $T(p, w(C(e)))$, and clearly $p_{1} \leq T(p, w(C(e))) \leq p_{3}$. Thus, we can define a voting game by $\Gamma=(Z, f, E, u, g, T)$, and our equilibrium concept is the Aumann (1959) SNE.

Definition 3.2 A strategy profile e $\in E$ is a strong Nash equilibrium (SNE) of the voting game $\Gamma$ if there exists no (measurable subset) $D \subseteq Z$ and no other strategy profile $e^{\prime} \in E$ such that $e_{x}^{\prime}=e_{x}$, for every $x \notin D$ and $u\left(T\left(p, w\left(C\left(e^{\prime}\right)\right)\right) ; \alpha\right)>$ $u(T(p, w(C(e))) ; \alpha)$, for every $\alpha \in D$.

To simplify some of the proofs we restrict our analysis to a particular family of subadditive functions $g$ :

${ }^{3}$ However, it is worth mentioning that Gomberg (2005) provides an example of non-existence for a three party case for any dimension of the policy space. 
Assumption 3.3 The function $g$ is given by

$$
g^{i}(w)=\frac{h\left(w^{i}\right)}{h\left(w^{1}\right)+h\left(w^{2}\right)+h\left(w^{3}\right)}
$$

where the function $h$ is continuously differentiable when $w^{i}>0$, increasing and strictly concave, $h(0)=0, h(1)=1$, and $h^{\prime}(0)=\infty$.

An important example of such subadditive functions is given by $h(x)=x^{\gamma}$, $0<\gamma<1$.

Let the strategy profile $e^{*} \in E$ be a SNE. We shall show existence of SNE such that all party memberships are intervals. Thus, we can identify the partition ${ }^{4}$ $C\left(e^{*}\right)$ by the numbers, $a_{1}$ and $a_{2}, 0 \leq a_{1} \leq a_{2} \leq 1$ such that $C_{1}\left(e^{*}\right)=\left[0, a_{1}\right]$, $C_{2}\left(e^{*}\right)=\left[a_{1}, a_{2}\right]$ and $C_{3}\left(e^{*}\right)=\left[a_{2}, 1\right]$. Thus, for instance, the case in which party 2 gets no votes can be represented by $a_{1}=a_{2}$.

Since individual deviations from the equilibrium strategy $e^{*}$ do not change the outcome policy $T\left(p, w\left(C\left(e^{*}\right)\right)\right)$, we only need to analyze deviations by coalitions of positive measure.

The following lemma provides conditions that have to be satisfied by any strong Nash equilibrium with interval memberships in a three-party voting game with the outcome policy function satisfying the above conditions. In words, it states that the equilibrium policy outcome must coincide with the ideal policy of either the rightmost or the leftmost member of the centrist party (otherwise, there would always exist a sufficiently small subcoalition of voters with ideal points between the policy outcome and the membership partition point that could shift the outcome in their direction by switching parties). In addition, it requires that the ratio of the distances between the policy oucome and the policy proposals of one of the "extremist" and the centrist parties must be inversely related to the ratio of the rate with which these parties' influence grows with their vote share.

Lemma 3.4 Let Assumptions 3.1 and 3.3 hold. Then, the strategy profile $e^{*}$ is a $S N E$ iff either of the following conditions hold.

If $T\left(p, w\left(C\left(e^{*}\right)\right)\right) \geq p_{2}$, then $T\left(p, w\left(C\left(e^{*}\right)\right)\right)=a_{2}$ and

$$
\frac{T\left(p, w\left(C\left(e^{*}\right)\right)\right)-p_{1}}{T\left(p, w\left(C\left(e^{*}\right)\right)\right)-p_{2}}=\frac{h^{\prime}\left(w_{2}\right)}{h^{\prime}\left(w_{1}\right)}
$$

$$
\begin{array}{r}
\text { If } T\left(p, w\left(C\left(e^{*}\right)\right)\right) \leq p_{2} \text {, then } T\left(p, w\left(C\left(e^{*}\right)\right)\right)=a_{1} \text { and } \\
\frac{T\left(p, w\left(C\left(e^{*}\right)\right)\right)-p_{3}}{T\left(p, w\left(C\left(e^{*}\right)\right)\right)-p_{2}}=\frac{h^{\prime}\left(w_{2}\right)}{h^{\prime}\left(w_{3}\right)}
\end{array}
$$

\footnotetext{
${ }^{4}$ Note, that $C\left(e^{*}\right)$ defined in this way is not a partition of $Z$ in the usual, strict meaning of this word, since the elements of $C\left(e^{*}\right)$ intersect at the end points of the interval. However, since this intersections consist exactly of one point and, hence, have measure zero, we may ignore them. Alternatively, we might define the sets $C_{i}\left(e^{*}\right)$ using half-open intervals. Nevertheless, for simplicity of notation, we shall use the above definition with closed intervals.
} 
Proof We show first that if $e^{*}$ is a SNE, then either $T\left(p, w\left(C\left(e^{*}\right)\right)\right)=a_{1}$ or $T\left(p, w\left(C\left(e^{*}\right)\right)\right)=a_{2}$. Let $e^{*}$ be a SNE and suppose, for example, that $T\left(p, w\left(C\left(e^{*}\right)\right)\right)>a_{2}$ (the proof in cases $T\left(p, w\left(C\left(e^{*}\right)\right)\right)<a_{1}$ and $\left.a_{1}<T\left(p, w\left(C\left(e^{*}\right)\right)\right)<a_{2}\right)$ is similar $)$. Since $T\left(p, w\left(C\left(e^{*}\right)\right)\right)$ is a convex combination of $p_{1}, p_{2}$ and $p_{3}$ we know that $T\left(p, w\left(C\left(e^{*}\right)\right)\right) \leq p_{3}$ and it is straightforward to see that in equilibrium $T\left(p, w\left(C\left(e^{*}\right)\right)\right)<p_{3}$.

Let $0<k<T\left(p, w\left(C\left(e^{*}\right)\right)\right)-a_{2}$ and let $D$ be the interval $\left(a_{2}, a_{2}+k\right)$. Since the density $f$ is strictly positive, $D$ has positive measure. We shall show for some $0<k<T\left(p, w\left(C\left(e^{*}\right)\right)\right)-a_{2}$, the above set of agents, $D$, can deviate from the strategy profile $e^{*}$ and be better off.

Consider the strategy profile $e^{\prime} \in E$ such that $e_{\alpha}^{\prime}=e_{a}^{*}, \forall \alpha \notin D$ and $e_{\alpha}^{\prime}=$ $\left\{p_{1}\right\}, \forall \alpha \in D$. Since the function $T(p, w(C(e)))$ is continuous we can always make the set $D$ small enough so that $\left|T\left(p, w\left(C\left(e^{*}\right)\right)\right)-T\left(p, w\left(C\left(e^{\prime}\right)\right)\right)\right|<k$. In this case, if $T\left(p, w\left(C\left(e^{*}\right)\right)\right)>T\left(p, w\left(C\left(e^{\prime}\right)\right)\right)$ all the agents in the deviating coalition $D$ would be better off under the strategy profile $s^{\prime}$. Thus, it only remains to be proven that indeed

$$
T\left(p, w\left(C\left(e^{*}\right)\right)\right)>T\left(p, w\left(C\left(e^{\prime}\right)\right)\right) \quad \text { for } k \text { small enough }
$$

Recall that

$$
\begin{aligned}
T\left(p, w\left(C\left(e^{*}\right)\right)\right)= & p_{1} \frac{h\left(w_{1}\right)}{h\left(w_{1}\right)+h\left(w_{2}\right)+h\left(w_{3}\right)}+p_{2} \frac{h\left(w_{2}\right)}{h\left(w_{1}\right)+h\left(w_{2}\right)+h\left(w_{3}\right)} \\
& +p_{3} \frac{h\left(w_{3}\right)}{h\left(w_{1}\right)+h\left(w_{2}\right)+h\left(w_{3}\right)}
\end{aligned}
$$

It is convenient to write $I(w)=T(p, w)$, where $w=\left(w_{1}, w_{2}, w_{3}\right)$. A sufficient condition for condition 3.3 to hold true is

$$
\left.\frac{\mathrm{d} I\left(w_{1}+\varepsilon, w_{2}, w_{3}-\varepsilon\right)}{\mathrm{d} \varepsilon}\right|_{\varepsilon=0}<0
$$

Taking the derivative and rearranging terms, inequality 3.4 can be written as

$$
h^{\prime}\left(w_{3}\right)\left(I\left(w_{1}, w_{2}, w_{3}\right)-p_{3}\right)-h^{\prime}\left(w_{1}\right)\left(I\left(w_{1}, w_{2}, w_{3}\right)-p_{1}\right)<0
$$

Since $h^{\prime}>0$ and $I\left(w_{1}, w_{2}, w_{3}\right)<p_{3}$ and $I\left(w_{1}, w_{2}, w_{3}\right)>p_{1}$, inequality 3.5 holds and, for $k>0$ small enough, the coalition $D$ may profit by deviating to strategy $e^{*}$.

Next, we prove necessity for the case $T\left(p, w\left(C\left(e^{*}\right)\right)\right) \geq p_{2}$. The proof of the other alternative is analogous. Thus, from now until the end of the proof of Lemma 3.4, we assume that

$$
T\left(p, w\left(C\left(e^{*}\right)\right)\right) \geq p_{2}
$$

First of all, remark that, since $a_{1} \leq p_{2} \leq a_{2}$ then, from the above argument, we must have that $T\left(p, w\left(C\left(e^{*}\right)\right)\right)=a_{2}$. 
Necessity of 3.1: Let $C_{i}, i=1,2,3$, be the coalition of agents voting for the proposal $p_{i}$, and $w_{i}$ the corresponding vote share when agents follow the strategy profile $e^{*}$. Here we check the conditions needed to guarantee that:

(A) no coalition of agents in $C_{1}$ wants to deviate from $e^{*}$ and vote for proposal $p_{2}$ and

(B) no coalition of agents in $C_{2}$ wants to deviate from $e^{*}$ and vote for proposal $p_{1}$.

We deal with case A) first. Consider a coalition $D=[0, k] \subset C_{1}$ of size $\varepsilon$ that deviates from $e^{*}$ and votes for $p_{2}$. We choose $D$ so that $\varepsilon$ is small enough and, by continuity, the new outcome $T(p, w(C(e)))$ is as close as needed to $T\left(p, w\left(C\left(e^{*}\right)\right)\right)$ and $T(p, w(C(e))) \notin D$. Let us write $T\left(p, w\left(C\left(e^{*}\right)\right)\right) \equiv$ $I\left(w_{1}, w_{2}, w_{3}\right)$. We need to check that for "small" $\varepsilon$

$$
I\left(w_{1}, w_{2}, w_{3}\right) \leq I\left(w_{1}-\varepsilon, w_{2}+\varepsilon, w_{3}\right)
$$

If inequality 3.6 holds all the members in $D$ are (weakly) worse off by deviating from $e^{*}$. Thus, a necessary condition for $e^{*}$ to be a SNE is that

$$
\left.\frac{\mathrm{d} I\left(w_{1}-\varepsilon, w_{2}+\varepsilon, w_{3}\right)}{\mathrm{d} \varepsilon}\right|_{\varepsilon=0} \geq 0
$$

We have that

$$
\begin{aligned}
I\left(w_{1}, w_{2}, w_{3}\right)= & p_{1} \frac{h\left(w_{1}\right)}{h\left(w_{1}\right)+h\left(w_{2}\right)+h\left(w_{3}\right)}+p_{2} \frac{h\left(w_{2}\right)}{h\left(w_{1}\right)+h\left(w_{2}\right)+h\left(w_{3}\right)} \\
& +p_{3} \frac{h\left(w_{3}\right)}{h\left(w_{1}\right)+h\left(w_{2}\right)+h\left(w_{3}\right)}
\end{aligned}
$$

Taking the derivative and arranging terms we can write Eq. 3.7 as

$$
h^{\prime}\left(w_{1}\right)\left(T\left(p, w\left(C\left(e^{*}\right)\right)\right)-p_{1}\right)-h^{\prime}\left(w_{2}\right)\left(T\left(p, w\left(C\left(e^{*}\right)\right)\right)-p_{2}\right) \geq 0
$$

Thus, inequality 3.8 is a necessary condition for a SNE.

Next we deal with case (B). Consider a coalition $D=\left[a_{1}, k\right] \subset C_{2}$ of size $\varepsilon$ that deviates from $e^{*}$ and vote for $p_{1}$. We choose $D$ so that $\varepsilon$ is small enough and, by continuity, the new outcome $T(p, w(C(e)))$ is as close as needed to $T\left(p, w\left(C\left(e^{*}\right)\right)\right)$ and $T(p, w(C(e))) \notin D$. We need to check that for "small" $\varepsilon$

$$
I\left(w_{1}, w_{2}, w_{3}\right) \leq I\left(w_{1}+\varepsilon, w_{2}-\varepsilon, w_{3}\right)
$$

If equality 3.9 holds all the members in $D$ are (weakly) worse off by deviating from $e^{*}$. Thus, a necessary condition for $e^{*}$ to be a SNE is that

$$
\left.\frac{\mathrm{d} I\left(w_{1}+\varepsilon, w_{2}-\varepsilon, w_{3}\right)}{\mathrm{d} \varepsilon}\right|_{\varepsilon=0} \geq 0
$$


Taking the derivative and arranging terms we can write Eq. 3.10 as

$$
h^{\prime}\left(w_{1}\right)\left(T\left(p, w\left(C\left(e^{*}\right)\right)\right)-p_{1}\right)-h^{\prime}\left(w_{2}\right)\left(T\left(p, w\left(C\left(e^{*}\right)\right)\right)-p_{2}\right) \leq 0
$$

Thus, inequalities 3.11 and 3.8 have both to be satisfied. Hence, equality 3.1 in the statement of the Lemma has to hold.

Sufficiency of 3.1: The argument in the previous part of the proof shows that, if Equation 3.1 holds, no "small" coalition of $C_{1}$ wants to deviate and vote for $p_{2}$ and no "small" coalition in $C_{2}$ wants to deviate and vote for $p_{1}$. It remains to be proved that there are no additional profitable deviations from the strategy profile $e^{*}$. We first note that:

(i) No coalition formed by agents on different sides of $T\left(p, w\left(C\left(e^{*}\right)\right)\right.$ can deviate from $e^{*}$ so that all of its members would be better off.

(ii) Say that coalition $D \subset C_{i}$ deviates from $e^{*}$ and some of its members vote for $p_{j}$ and the remaining members vote for $p_{k}, i \neq j, k$ and $j \neq k$. Say that such deviation "moves" $T(p, w(C(e))$ in the direction to make all members of $D$ better off. Then, it is easy to show that there is a deviating coalition $D^{\prime} \subseteq D$ that can also achieve an improvement for all its members by voting either to $p_{j}$ or to $p_{k}$. Therefore, if we can show that coalitional deviations to a single party are impossible, we will have also shown the same about deviations in favor of different parties.

(iii) If no "small" subcoalition of $C_{1}$ (respectively, $C_{2}$ ) wants to deviate and vote for $p_{2}$ (respectively, $p_{1}$ ) then no subcoalition of any size of $C_{1}$ (respectively, $C_{2}$ ) wants to deviate and vote for $p_{2}$ (respectively, $p_{1}$ ). Thus, the necessary conditions analyzed in the first part of the proof also apply for coalition deviations of any size. To see it, consider the case of a deviation by a coalition of agents $D \subset C_{1}$. Let $\varepsilon$ be the size of such coalition. We have already proven that for $\varepsilon>0$ small enough $I\left(w_{1}-\varepsilon, w_{2}+\varepsilon, w_{3}\right)>I\left(w_{1}, w_{2}, w_{3}\right)$. We need to show now that such inequality holds for any size $\varepsilon$. Suppose not. So, for some $\varepsilon^{\prime}$ we have $I\left(w_{1}-\varepsilon^{\prime}, w_{2}+\varepsilon^{\prime}, w_{3}\right)<I\left(w_{1}, w_{2}, w_{3}\right)$. Since, $I\left(w_{1}-\varepsilon, w_{2}+\varepsilon, w_{3}\right)$ is continuous in $\varepsilon$ and

$$
\left.\frac{\mathrm{d} I\left(w_{1}-\varepsilon, w_{2}+\varepsilon, w_{3}\right)}{\mathrm{d} \varepsilon}\right|_{\varepsilon=0}>0
$$

there must exist a coalition of size $\bar{\varepsilon}<\varepsilon^{\prime}$ such that $I\left(w_{1}-\bar{\varepsilon}, w_{2}+\bar{\varepsilon}, w_{3}\right)=$ $I\left(w_{1}, w_{2}, w_{3}\right)$ and

$$
\left.\frac{\mathrm{d} I\left(w_{1}-\bar{\varepsilon}, w_{2}+\bar{\varepsilon}, w_{3}\right)}{\mathrm{d} \varepsilon}\right|_{\varepsilon=\bar{\varepsilon}} \leq 0
$$

We saw in part (1) that this inequality can be written as

$$
\frac{I\left(w_{1}-\bar{\varepsilon}, w_{2}+\bar{\varepsilon}, w_{3}\right)-p_{1}}{I\left(w_{1}-\bar{\varepsilon}, w_{2}+\bar{\varepsilon}, w_{3}\right)-p_{2}} \leq \frac{h^{\prime}\left(w_{2}+\varepsilon\right)}{h^{\prime}\left(w_{1}-\varepsilon\right)}
$$


But, this inequality together with

$$
\frac{I\left(w_{1}-\bar{\varepsilon}, w_{2}+\bar{\varepsilon}, w_{3}\right)-p_{1}}{I\left(w_{1}-\bar{\varepsilon}, w_{2}+\bar{\varepsilon}, w_{3}\right)-p_{2}}=\frac{I\left(w_{1}, w_{2}, w_{3}\right)-p_{1}}{I\left(w_{1}, w_{2}, w_{3}\right)-p_{2}}=\frac{h^{\prime}\left(w_{2}\right)}{h^{\prime}\left(w_{1}\right)}
$$

implies that

$$
\frac{h^{\prime}\left(w_{2}\right)}{h^{\prime}\left(w_{1}\right)} \leq \frac{h^{\prime}\left(w_{2}+\varepsilon\right)}{h^{\prime}\left(w_{1}-\varepsilon\right)}
$$

which contradicts the strict concavity of $h$.

The same argument works to show that it is enough to check deviations of "small" coalitions of agents. Thus, it is sufficient to analyze the possibility of the following kinds of deviations by (arbitrarily small-size) coalitions:

(a) agents in $C_{1}$ that deviate and vote for $p_{3}$

(b) agents in $C_{2}$ that deviate and vote for $p_{3}$

(c) agents in $C_{1} \cup C_{2}$ that deviate and vote for $p_{3}$

(d) agents in $C_{3}$ that deviate and vote for $p_{1}$

(e) agents in $C_{3}$ that deviate and vote for $p_{2}$

We start with case (a). If there is an (arbitrarily-small) coalition $D \in C_{1}$ that by voting for proposal $p_{3}$ improves the utility of all its members, then it must be that $I\left(w_{1}-\varepsilon, w_{2}, w_{3}+\varepsilon\right)<I\left(w_{1}, w_{2}, w_{3}\right)$. Taking the derivative with respect to $\varepsilon$ evaluated at $\varepsilon=0$, and rearranging terms, the previous inequality implies $\left(I\left(w_{1}, w_{2}, w_{3}\right)-p_{1}\right) h^{\prime}\left(w_{1}\right) \leq\left(I\left(w_{1}, w_{2}, w_{3}\right)-p_{3}\right) h^{\prime}\left(w_{3}\right)$. Since $I\left(w_{1}, w_{2}, w_{3}\right)=$ $a_{2} \in\left(p_{1}, p_{3}\right)$, we have $I\left(w_{1}, w_{2}, w_{3}\right)>p_{1}$ and $I\left(w_{1}, w_{2}, w_{3}\right)<p_{3}$, and $h^{\prime}>0$. Hence, the last inequality cannot hold true. The argument in case (d) is symmetric.

Almost the same argument works for cases (b) and (e). Indeed if such a coalitional deviation in (b) were possible it must have been that $I\left(w_{1}, w_{2}-\varepsilon, w_{3}+\varepsilon\right)<$ $I\left(w_{1}, w_{2}, w_{3}\right)$ (since moving the outcome rightwards of $a_{2}$ would make everyone in $C_{2}$ worse off $)$ and $\left(I\left(w_{1}, w_{2}, w_{3}\right)-p_{2}\right) h^{\prime}\left(w_{2}\right) \leq\left(I\left(w_{1}, w_{2}, w_{3}\right)-p_{3}\right) h^{\prime}\left(w_{3}\right)$, which by the same logic can be seen to be impossible since $I\left(w_{1}, w_{2}, w_{3}\right)=$ $a_{2} \in\left(p_{2}, p_{3}\right)$. A similar argument applies to case (c) as well (all agents in any subcoalition of $C_{1} \cup C_{2}$ can be better off only if the outcome moves to the left).

As mentioned above, the proof of the sufficiency of condition 3.2 is similar and we omit it.

It follows from the previous Proposition that in any SNE, the outcome policy has to coincide with the ideal policy for an agent "indifferent" between two of the proposals.

We next state the main result of this section

Lemma 3.5 Let Assumptions 3.1 and 3.3 hold. Then, there exists a SNE.

Proof Take an arbitrary number $a \in\left[p_{2}, p_{3}\right]$. Consider the following strategy profile $e^{a} \in E$. All the agents $\alpha>a$ play the strategy $e_{\alpha}^{a}=\left\{p_{3}\right\}$. The agents 
$\alpha \leq a$ are divided in two groups. The first group consists of all agents with types in $\left[0, s^{a}\right)$ and the second group is given by the set of agents with types in $\left[s^{a}, a\right]$. Any agent $\alpha \in\left[0, s^{a}\right)$ plays the strategy $e_{\alpha}^{a}=\left\{p_{1}\right\}$, and any agent $\alpha \in\left[s^{a}, a\right]$ plays the strategy $e_{\alpha}^{a}=\left\{p_{2}\right\}$. Let $w_{1}(a) \equiv F\left(s^{a}\right)$ (respectively, $w_{2}(a) \equiv F(a)-F\left(s^{a}\right)$ ) be the mass of agents in the first group (respectively, the mass of agents in the second group). The number $s^{a}$ is given by the solution to

$$
\frac{a-p_{1}}{a-p_{2}}=\frac{h^{\prime}\left(w_{2}(a)\right)}{h^{\prime}\left(w_{1}(a)\right)}=\frac{h^{\prime}\left(F(a)-F\left(s^{a}\right)\right)}{h^{\prime}\left(F\left(s^{a}\right)\right)}
$$

(Notice that Eq. 3.12 looks similar to the first equation in 3.1). Next we show that $s^{a}$ is well defined and Eq. 3.12 always has a solution. Since $a>p_{2}>p_{1}$ the first term in Eq. 3.12 is a (finite) positive number. And, since the functions $h^{\prime}$ and $F$ are continuous, so is

$$
\frac{h^{\prime}\left(F(a)-F\left(s^{a}\right)\right)}{h^{\prime}\left(F\left(s^{a}\right)\right)}
$$

When $s^{a}$ approaches 0 , the term $h^{\prime}\left(F\left(s^{a}\right)\right)$ approaches $\infty$ and

$$
\frac{h^{\prime}\left(F(a)-F\left(s^{a}\right)\right)}{h^{\prime}\left(F\left(s^{a}\right)\right)}
$$

approaches 0 . When $s^{a}$ approaches $a$, we have that

$$
\frac{h^{\prime}\left(F(a)-F\left(s^{a}\right)\right)}{h^{\prime}\left(F\left(s^{a}\right)\right)}
$$

approaches infinity. By continuity of $\frac{h^{\prime}\left(F(a)-F\left(s^{a}\right)\right)}{h^{\prime}\left(F\left(s^{a}\right)\right)}$, it follows that there is a solution, $0<s^{a}<a$ to Eq. 3.12.

Let $I(a) \equiv T\left(p, w\left(C\left(e^{a}\right)\right)\right)$ be the outcome policy when all agents follow the strategy profile $e^{a}$. Notice that $I(a)$ is continuous on the interval $\left(p_{2}, p_{3}\right)$.

If we can find some $a^{*} \in\left[p_{2}, p_{3}\right]$ such that $I\left(a^{*}\right)=a^{*}$, then, the strategy profile $e^{a^{*}}$ is a SNE, because: (i) equality 3.12 is equivalent to the second equality in the statement of Lemma 3.4 and, (ii) the first equality in the statement of Lemma 3.4 also holds since $a_{2}=a^{*}=T\left(p, w\left(C\left(e^{a^{*}}\right)\right)\right)$. There are three cases to analyze:

(a) Consider first the case that $I\left(p_{2}\right)>p_{2}$. Since, $I(a) \leq p_{3}$ for every $a \in$ $\left[p_{2}, p_{3}\right]$ and $I$ is decreasing in $a$ (because $w_{3}(a)=1-F(a)$ is decreasing in $a)$, a standard continuity argument shows that there is some $a^{*} \in\left[p_{2}, p_{3}\right]$ such that $I\left(a^{*}\right)=a^{*}$.

(b) Consider now the possibility that $I\left(p_{2}\right)<p_{2}$. In this case, since $I$ is decreasing, we also have that $I\left(a^{\prime}\right)<p_{2}$ for every $a^{\prime} \in\left[p_{2}, a\right]$. Consider now the function $\widehat{I}(a)$ that is defined in a similar way to $I(a)$ but changing the coalitions on each side of $a$. Namely, all the agents $\alpha<a$ play the strategy 
$\widehat{e}_{\alpha}^{a}=\left\{p_{1}\right\}$. The agents $\alpha>a$ are divided in two groups. The first group consists of all agents in $\left[a, s^{a}\right)$ and the second group is given by $\left[s^{a}, 1\right]$. Agents in the first group vote for $p_{2}$ and agents in the second group vote for $p_{3}$. Now we have $w_{2}(a) \equiv F\left(s^{a}\right)-F(a)$ and $w_{3}(a) \equiv 1-F\left(s^{a}\right)$. The number $s^{a}$ is given by the solution to

$$
\frac{a-p_{2}}{a-p_{3}}=\frac{h^{\prime}\left(w_{3}(a)\right)}{h^{\prime}\left(w_{2}(a)\right)}
$$

One can easily show that

$$
\lim _{a \rightarrow p_{2}} I(a)=\widehat{I}\left(p_{2}\right)
$$

Thus, $\widehat{I}\left(p_{2}\right)<p_{2}$ and we have a "symmetric" situation to the one faced in case a). Thus, the same continuity argument can be used here to prove that there exists $a \leq p_{2}$ such that $\widehat{I}(a)=a$ and the corresponding strategy profile $\hat{e}^{a}$ is a SNE.

(c) Otherwise, we have that

$$
\lim _{a \rightarrow p_{2}} I(a)=p_{2}
$$

It is clear that in this case there is a SNE consisting on everybody on $\left[0, p_{2}\right]$ voting for $p_{1}$ and everybody on $\left[p_{2}, 1\right]$ voting for $p_{3}$. Notice that the conditions on Lemma 3.4 are satisfied since

$$
\frac{a-p_{1}}{a-p_{2}}=\infty, \quad \frac{h^{\prime}\left(w_{2}(a)\right)}{h^{\prime}\left(w_{1}(a)\right)}=\infty
$$

and the outcome is $T(p, w(C(e)))=a_{1}=a_{2}=p_{2}$.

Remark 3.6 Notice that the previous Lemma does not rule out the possibility that in all SNE only two parties receive a positive mass of vote. However, if $w_{2}=0$, i.e. the second party receives no positive measure of votes, then we must have that $h^{\prime}\left(w_{2}\right)=+\infty$. And, in order for Eqs. 3.1 and 3.2 to hold, we must have that

$$
T\left(p, w\left(C\left(e^{*}\right)\right)\right)=p_{2}=a_{1}=a_{2}
$$

An example might help to clarify this possibility.

Example 3.7 Consider the proposals $p_{1}=0, p_{2}=1 / 2$ and $p_{3}=1$. Suppose that the distribution of agents on $Z=[0,1]$ is uniform. It is not difficult to show that $C_{1}=[0,1 / 2), C_{2}=\{\varnothing\}$ and $C_{3}=[1 / 2,1]$, correspond to a SNE such that $T\left(p, w\left(C\left(e^{*}\right)\right)\right)=1 / 2$. Consider any coalition of agents $D \subset C_{1}$. They can deviate from $e^{*}$ by voting either to $p_{2}$ or to $p_{3}$. If they vote for 
$p_{3}$ the weight of such policy increases and policy $p_{2}$ reduces its weight. Thus, the outcome $T\left(p, w\left(C\left(e^{\prime}\right)\right)\right)$ clearly moves to the right of $T\left(p, w\left(C\left(e^{*}\right)\right)\right)$, and all the agents in $D$ are worse off. If they vote for $p_{2}$ the argument is a little bit more involved. As in the proof of Lemma 3.4 it is convenient to write $T(p, w(C(e))) \equiv I\left(w_{1}, w_{2}, w_{3}\right)$, where $w_{2}=0$. We want to show that the derivative $\left.\frac{\mathrm{d} I\left(w_{1}-\varepsilon, 0+\varepsilon, w_{3}\right)}{\mathrm{d} \varepsilon}\right|_{\varepsilon=0} \geq 0$. One can easily show that such inequality holds iff $p_{2} h^{\prime}\left(w_{2}\right) \geq I\left(w_{1}, w_{2}, w_{3}\right)\left(h^{\prime}\left(w_{2}\right)-h^{\prime}\left(w_{1}\right)\right)$. And since $p_{2}=I\left(w_{1}, w_{2}, w_{3}\right)$ and $h^{\prime} \geq 0$ the inequality holds.

Remark 3.8 The proof of Lemma 3.5 shows that, given any proposal profile $p=\left(p_{1}, p_{2}, p_{3}\right)$ with $p_{1} \leq p_{2} \leq p_{3}$, it is possible to construct a SNE voter partition $C(p)=\left(C_{1}(p), C_{2}(p), C_{3}(p)\right)$ so that the $C_{i}(p)$ 's are intervals, whose end points vary continuously with $p$.

\section{Definition of multiparty equilibrium}

As stated above, the objective of this paper is to analyze the issue of existence of an overall equilibrium where the membership of parties, their policy platforms, as well as the voting behavior of agents are endogenous. To do this we first establish conditions ensuring that the outcome of the voting game obtained when party platforms are exogenously fixed is well-defined. These conditions, of course, depend on whether voters behave sincerely or strategically. Once we understand the conditions guaranteeing existence of equilibrium in the voting game we can tackle the problem of existence of an overall equilibrium with endogenous party platforms.

The way the game is modeled is based on the idea that the internal and the external politics of parties are interrelated, in the sense that party membership determines policy platforms and policy platforms serve to attract citizens to parties. Assuming that party membership coincides with party electorate (an assumption that may be relaxed) we can say that equilibrium obtains when the voting partition resulting from a policy profile on offer induces the same policy profile.

It is easy to construct equilibria with parties being identical in their policy positions. In this case any voting pattern corresponds trivially to an equilibrium of the voting game, and, therefore, we are free to chose a population partition to support the identical party positions. Given the apparent "pluralism" of positions on policy issues observed in most political systems, it is, however, of interest to study existence of equilibria with non-identical parties.

Definition 4.1 Given an outcome function $T$ and a party policy mapping $P$, we say that $\left(p^{*}, C^{*}\right)$ is a multi-party equilibrium if:

(i) $p^{*}=P\left(C^{*}\right)$

(ii) $C^{*}$ is an equilibrium of the voting game induced by $p^{*}$.

If, furthermore, the equilibrium party proposals are distinct $\left(p^{j *} \neq p^{k *}\right.$ for $j$, $k=1, \ldots, m, j \neq k$ ) such equilibrium is called pluralistic. 
Given a multi-party equilibrium $\left(p^{*}, C^{*}\right)$, the associated policy outcome is $T^{*}=T\left(p^{*}, w\left(C^{*}\right)\right)$.

\section{Existence of multi-party equilibrium with sincere voters}

Let $p=\left(p_{1}, \ldots, p_{m}\right) \in Z$ be the policies proposed by the parties. In the following, it will be convenient to use the following notation

$$
a_{i}(p)= \begin{cases}\frac{p_{i}+p_{i+1}}{2} & \text { if } i=1, \ldots, m-1 \\ 0 & \text { if } i=0 \\ 1 & \text { if } i=m .\end{cases}
$$

and also $p_{0}=0, p_{m+1}=1$. We also let

$$
a(p)=\left(a_{0}(p), \ldots, a_{m}(p)\right)
$$

Recall that for each $i=1, \ldots, m$, the set $C_{i}(p)$ consists of those agents that vote for proposal $p_{i}$. In the case in which agents vote sincerely, we have that, with the convention above,

$$
C_{i}(p)=\left[a_{i-1}(p), a_{i}(p)\right]
$$

Let $X$ be set of possible $m$ parties and let $Y$ be the set of possible $m$ policies. We may identify these sets with

$$
\begin{aligned}
X & =\left\{a=\left(a_{1}, \ldots, a_{m-1}\right) \in Z^{m-1}: a_{1}<a_{2}<\cdots<a_{m-1}\right\} \\
Y & =\left\{p=\left(p_{1}, \ldots, p_{m}\right) \in Z^{m}: p_{1}<p_{2}<\cdots<p_{m}\right\}
\end{aligned}
$$

An element $a \in X$ can be identified with a partition $C=\left(C_{1}, \ldots, C_{m}\right)$ of $Z$ into intervals in the obvious way. That is, $C_{i}=\left[a_{i-1}, a_{i}\right]$, for $i=1, \ldots, m$. Abusing the notation, we will write $P(a)=P(C)$, whenever $C$ and $a$ are related in such a manner. Note that, since $a_{i-1}<P_{i}\left(a_{1}, \ldots, a_{m-1}\right)<a_{i}$, for each $i=1, \ldots, m-1$, we have that $P: X \rightarrow Y$ and $C: Y \rightarrow X$. In this context, an equilibrium corresponds to a fixed point of the mapping $\varphi=P \circ C: Y \rightarrow Y$. Thus, to prove the existence of equilibrium, it is enough that we restrict ourselves to parties given by intervals.

The next assumption requires the continuity of $P$, when restricted to this set

Assumption 5.1 The mapping $P: X \rightarrow Y$ is continuous.

Clearly, $C: Y \rightarrow X$ is continuous, and hence so is the mapping $\varphi: X \rightarrow X$. Now, we are ready to state our existence Theorem for the case in which agents vote sincerely. The following result guarantees not only that there is an equilibrium, but also that the policies proposed by the parties are different. For the case of sincere voting, this entails that, in equilibrium each party receives a positive amount of votes. 
Theorem 5.2 Under Assumptions 2.1, 2.2 and 5.1 there exists a pluralistic equilibrium.

Proof We first state a preliminary result. It is a direct consequence of Assumption 2.2 and it guarantees that the policies are uniformly bounded away from the boundary of the proposing party.

Lemma 5.3 There is some $\eta>0$ such that for every $a=\left(a_{1}, \ldots, a_{m-1}\right) \in X$ and every $i=0, \ldots, m$, we have that

$$
\left.\min \left\{P_{i}(a)-a_{i-1}, a_{i}-P_{i}(a)\right)\right\} \geq \eta\left(F\left(a_{i}\right)-F\left(a_{i-1}\right)\right)
$$

Proof Suppose the lemma does not hold. Then, there is some $i=0, \ldots, m$ and a sequence $a^{n}=\left(a_{1}^{n}, \ldots, a_{m-1}^{n}\right) \in X$ such that for each $n=1,2, \ldots$ we have that

$$
\min \left\{P_{i}\left(a^{n}\right)-a_{i-1}^{n}, a_{i}^{n}-P_{i}\left(a^{n}\right)\right\} \leq \frac{F\left(a_{i}^{n}\right)-F\left(a_{i-1}^{n}\right)}{n} \leq \frac{1}{n}
$$

Let us use the shorter the notation $p_{i}^{n}=P_{i}\left(a^{n}\right)$. By compactness and taking subsequences if necessary, we may assume that $\left\{a_{i-1}^{n}\right\}_{n=1}^{\infty}$ converges to, say $a_{i-1} \in[0,1]$. By taking further subsequences, if needed, we may also assume that, either

$$
p_{i}^{n}-a_{i-1}^{n} \leq \frac{F\left(a_{i}^{n}\right)-F\left(a_{i-1}^{n}\right)}{n} \text { for all } n=1,2, \ldots
$$

or else,

$$
a_{i}^{n}-p_{i}^{n} \leq \frac{F\left(a_{i}^{n}\right)-F\left(a_{i-1}^{n}\right)}{n} \text { for all } n=1,2, \ldots
$$

Let us suppose that the first case holds, the alternative case being completely analogous. First, note that the sequence $p_{i}^{n}$ also converges to $a_{i-1} \mathrm{By}$ Assumption 2.2, we must have that for any $y \in C_{i}^{n}=\left[a_{i-1}^{n}, a_{i}^{n}\right]$,

$$
\mu\left(\left\{\alpha \in C_{i}^{n}: u\left(p_{i}^{n}, \alpha\right) \geq u(y, \alpha)\right\}\right) \geq \delta \mu\left(C_{i}^{n}\right)=\delta\left(F\left(a_{i}^{n}\right)-F\left(a_{i-1}^{n}\right)\right)
$$

We claim that this implies the inequality

$$
F\left(p_{i}^{n}\right)-F\left(a_{i-1}^{n}\right) \geq \delta\left(F\left(a_{i}^{n}\right)-F\left(a_{i-1}^{n}\right)\right)
$$

Otherwise, suppose

$$
F\left(p_{i}^{n}\right)-F\left(a_{i-1}^{n}\right)+\varepsilon \leq \delta\left(F\left(a_{i}^{n}\right)-F\left(a_{i-1}^{n}\right)\right)
$$


for some $\varepsilon>0$. Then, for every $y \in\left(p_{i}^{n}, a_{i}^{n}\right)$ we have that

$$
\mu\left(\left\{\alpha \in C_{i}^{n}: u\left(p_{i}^{n}, \alpha\right) \geq u(y, \alpha)\right\}\right)=F\left(\frac{p_{i}^{n}+y}{2}\right)-F\left(a_{i-1}^{n}\right)
$$

And, since $F$ is continuous, by taking $y$ close enough to $p_{i}^{n}$ we have that

$\mu\left(\left\{\alpha \in C_{i}^{n}: u\left(p_{i}^{n}, \alpha\right) \geq u(y, \alpha)\right\}\right)<F\left(p_{i}^{n}\right)-F\left(a_{i-1}^{n}\right)+\varepsilon \leq \delta\left(F\left(a_{i}^{n}\right)-F\left(a_{i-1}^{n}\right)\right)$

which contradicts Eq. 5.2. Hence, Eq. 5.3 holds and we have that

$$
p_{i}^{n}-a_{i-1}^{n}<\frac{F\left(a_{i}^{n}\right)-F\left(a_{i-1}^{n}\right)}{n} \leq \frac{F\left(p_{i}^{n}\right)-F\left(a_{i-1}^{n}\right)}{n \delta}
$$

So,

$$
\frac{F\left(p_{i}^{n}\right)-F\left(a_{i-1}^{n}\right)}{p_{i}^{n}-a_{i-1}^{n}} \geq n \delta
$$

Taking limits as $n \rightarrow \infty$, we obtain that $f\left(a_{i-1}+\right)=F^{\prime}\left(a_{i-1}+\right)=+\infty$, which contradicts that $f$ is continuous and the lemma is proved.

Let $\varepsilon>0$ "small enough" ( $\varepsilon$ will be determined later) and consider the domain

$$
\Sigma=\left\{\left(p_{1}, \ldots, p_{m}\right) \in Y:\left|p_{i}-p_{j}\right| \geq \varepsilon \text { for all } 0 \leq i<j \leq m+1\right\}
$$

Recall that $p_{0}=0, p_{m+1}=1$. Of course, $\Sigma$ depends on $\varepsilon$. Nevertheless, to avoid cluttering the notation we will not write this dependency explicitly.

The function $\varphi$ maps $\Sigma$ into $Y$. We shall construct a new "truncated" mapping $\bar{\varphi}=\left(\bar{\varphi}_{1}, \ldots, \bar{\varphi}_{m}\right)$ defined by

$$
\bar{\varphi}_{i}(p)= \begin{cases}a_{i-1}(p)+\frac{\varepsilon}{2} & \text { if } a_{i-1}(p) \leq \varphi_{i}(p) \leq a_{i-1}(p)+\frac{\varepsilon}{2} \\ \varphi_{i}(p) & \text { if } a_{i-1}(p)+\frac{\varepsilon}{2} \leq \varphi_{i}(p) \leq a_{i}(p)-\frac{\varepsilon}{2} \\ a_{i}(p)-\frac{\varepsilon}{2} & \text { if } a_{i}(p)-\frac{\varepsilon}{2} \leq \varphi_{i}(p) \leq a_{i}(p)\end{cases}
$$

where, for $i=1, \ldots, m-1$, the term $a_{i}(p)$, defined in Equation 5.1, is the ideal policy of the voter sincerely indifferent between $p_{i}$ and $p_{i+1}$. Again, we will not write explicitly the dependency of $\bar{\varphi}$ on $\varepsilon$.

The mapping $\bar{\varphi}: \Sigma \rightarrow \Sigma$ is a continuous mapping defined on a compact, simply connected subset of $\mathbb{R}^{m}$, that agrees with $\varphi$ whenever $\varphi$ 's image is inside $\Sigma$. By Brouwer's Fixed Point Theorem, there is some $p^{*} \in Y$ such that $\bar{\varphi}\left(p^{*}\right)=p^{*}$.

Without loss of generality, we may assume that $\eta$, as determined in Lemma 5.3, is less than 1. To finish the proof of Theorem 5.2, it is enough to show that $\bar{\varphi}\left(p^{*}\right)=\varphi\left(p^{*}\right)$ if $\varepsilon$ is small enough. This is basically the content of the next Lemma. It states that fixed points of the map $\bar{\varphi}$ cannot be "too close". 
Let

$$
N=\min \left\{1, \min _{x \in A} f(x)\right\}
$$

and note that $0<N \leq 1$.

Lemma 5.4 Suppose $\varepsilon>0$ is such that

$$
\frac{\varepsilon}{2 N^{m-1} \eta^{m-1}}<\frac{1}{m}
$$

and suppose $p \in \Sigma$ satisfies $\bar{\varphi}(p)=p$. Then, $\bar{\varphi}(p)=\varphi(p)$.

Proof Let $\varepsilon>0$ and suppose $p \in \Sigma$ verifies $\bar{\varphi}(p)=p$. By assumption, $p_{i+1}-p_{i} \geq$ $\varepsilon$ for every $i=1, \ldots, m$. Hence,

$$
a_{i}(p)+\frac{\varepsilon}{2} \leq p_{i+1} \leq a_{i+1}(p)-\frac{\varepsilon}{2}
$$

for every $i=1, \ldots, m$.

We divide the sets of indices $\{1, \ldots, m\}$ into two sets,

$$
\begin{aligned}
& M_{1}=\left\{k=1, \ldots, m: p_{k}=\bar{\varphi}_{k}(p)=\varphi_{k}(p)\right\} \\
& M_{2}=\left\{j=1, \ldots, m: p_{j}=\bar{\varphi}_{j}(p) \neq \varphi_{j}(p)\right\}
\end{aligned}
$$

Suppose now that $\bar{\varphi}(p) \neq \varphi(p)$. That is, $M_{2} \neq \emptyset$. Then, for those indices $j \in M_{2}$, we have that one of the inequalities

$$
a_{j-1}(p)+\frac{\varepsilon}{2} \leq \varphi_{j}(p) \leq a_{j}(p)-\frac{\varepsilon}{2}
$$

does not hold. In either case, it follows that

$$
\left.\min \left\{\varphi_{j}(p)-a_{j-1}(p), a_{j}(p)-\varphi_{j}(p)\right)\right\} \leq \frac{\varepsilon}{2}
$$

and, from Lemma 5.3, we see that (shortening the notation to $a_{j}=a_{j}(p)$ ),

$$
\eta\left(F\left(a_{j}\right)-F\left(a_{j-1}\right)\right) \leq \frac{\varepsilon}{2}
$$

On the other hand,

$$
F\left(a_{j}\right)-F\left(a_{j-1}\right)=\int_{a_{j-1}}^{a_{j}} f(t) \mathrm{d} t \geq N\left(a_{j}-a_{j-1}\right)
$$


so,

$$
a_{j}-a_{j-1} \leq \frac{\varepsilon}{2 \eta N}
$$

Let us now consider an index $1 \leq k \leq m$ such that $p_{k}=\bar{\varphi}_{k}(p)=\varphi_{k}(p)$ and suppose that the interval $C_{k}(p)=\left[a_{k-1}(p), a_{k}(p)\right]$ is adjacent to an interval for which we have a bound as in equation 5.5. For the sake of concreteness, let us consider the case in which $k=i+1$ with

$$
a_{i}-a_{i-1} \leq \alpha(i)
$$

for some $\alpha(i)>0$. We remark that,

$$
p_{i+1}-a_{i}=a_{i}-p_{i} \leq a_{i}-a_{i-1} \leq \alpha(i)
$$

On the other hand, since $\varphi_{i+1}(p)=p_{i+1}$ and applying again Lemma 5.3 we see that

$$
p_{i+1}-a_{i}=\varphi_{i+1}(p)-a_{i} \geq \eta\left(F\left(a_{i+1}\right)-F\left(a_{i}\right)\right)=\eta \int_{a_{i}}^{a_{i+1}} f(t) \mathrm{d} t \geq \eta N\left(a_{i+1}-a_{i}\right)
$$

Hence,

$$
a_{i+1}-a_{i} \leq \frac{\alpha(i)}{\eta N}
$$

Now starting from an index $j \in M_{2}$ and repeating successively the previous argument for the indices $k \in M_{2}$, after at most $m-1$ steps, we see that

$$
\max \left\{a_{i+1}-a_{i}: i=0, \ldots, m\right\} \leq \frac{\varepsilon}{2 \eta^{m-1} N^{m-1}}
$$

But then,

$$
1=\sum_{i=0}^{m}\left(a_{i+1}-a_{i}\right) \leq m \frac{\varepsilon}{2 \eta^{m-1} N^{m-1}}<1
$$

a contradiction. From this, Lemma 5.4 follows and, hence Theorem 5.2 is proved.

\section{Multiparty equilibrium with strategic voters and subadditivity}

We now prove an existence result for multiparty equilibria with strategic voters. For the rest of this section, the setting is the same as in Sect. 3. That is, we 
restrict ourselves to the case of three parties and a weight function given as in Assumption 3.3. Again, a similar existence result holds for more than three parties, but in such case the analysis becomes extremely cumbersome. Thus, for simplicity we restrict ourselves to the three-party case.

In particular, the theses in Lemmas 3.4 and 3.5 apply to our setting. Accordingly, we assume that agents behave strategically as described in Definition 3.2.

The map $C: Y \rightarrow X$ is now defined by $C(p)=\left(C_{1}(p), C_{2}(p), C_{3}(p)\right)$ with $C_{i}(p)=\left[a_{i-1}(p), a_{i}(p)\right]$ an interval whose end points are given by the functions $a_{i-1}(p), a_{i}(p)$, defined by Equation 5.1. Hence, They are continuous on the proposals $p=\left(p_{1}, p_{2}, p_{3}\right) \in Y$. We adopt the convention that $p_{0}=0$ and $p_{4}=1$. In case $p_{2}$ receives no votes, we will assume that $C_{2}(p)=\left\{p_{2}\right\}$.

As in the previous section, we define $\varphi: Y \rightarrow Y$ by $\varphi=P \circ C$. Now, we state the main result of this section.

Theorem 6.1 Under Assumptions 2.1,2.2 and 5.1 there exists a multiparty pluralistic equilibrium.

The proof parallels that of Theorem 5.2. We first prove the analogue of Lemma 5.4.

Lemma 6.2 Let Assumptions 2.1, 2.2 and 5.1 hold. Then, there is some $\eta>0$ such that if for some $p \in X$ and $i=0, \ldots, 4$ we have that $p_{i+1}-p_{i} \leq \eta$, then

(1) $\varphi_{1}(p)>p_{1}$ if $i=0$.

(2) $\varphi_{i}(p)<p_{i}$ and $\varphi_{i+1}(p)>p_{i+1}$ if $i=1,2$.

(3) $\varphi_{3}(p)<p_{3}$ if $i=3$.

Proof Suppose the Lemma does not hold. Then, for each $n=1,2 \ldots$ we can find $p^{n}=\left(p_{1}^{n}, p_{2}^{n}, p_{3}^{n}\right) \in X$ and $i_{n} \in\{0, \ldots, 4\}$ such that

$$
p_{i_{n}+1}^{n}-p_{i_{n}}^{n} \leq \frac{1}{n}
$$

and either $\varphi_{i_{n}}\left(p^{n}\right) \geq p_{i_{n}}^{n}$ or $\varphi_{i_{n}+1}\left(p^{n}\right) \leq p_{i_{n}+1}^{n}$.

By a standard compactness argument, we may assume all sequences above converge. And, since the index $i_{n} \in\{0, \ldots, 4\}$ takes only finitely many values, we may assume $i_{n}$ is constant for all $n=1,2 \ldots$. To fix ideas we assume $i_{n}=1$ for all $n=1,2 \ldots$ and $\varphi_{i_{n}+1}\left(p^{n}\right) \leq p_{i_{n}+1}^{n}$. The other cases can be handled by a similar argument.

Hence, w.l.o.g. we may assume that for all $n=1,2 \ldots$, the following hold,

$$
\begin{aligned}
p_{2}^{n}-p_{1}^{n} & \leq \frac{1}{n} \\
\varphi_{2}\left(p^{n}\right) & \leq p_{2}^{n} \\
\lim _{n \rightarrow \infty} p_{3}^{n} & =p_{3} \in Z
\end{aligned}
$$


Let us denote $C_{i}\left(p^{n}\right)=\left[a_{i-1}^{n}, a_{i}^{n}\right]$. Since $p_{1}^{n}<a_{1}^{n}<p_{2}^{n}$, we must have that the sequence $\left\{a_{1}^{n}\right\}_{n=1}^{\infty}$ converges to, say $a_{1}$, and also

$$
\lim _{n \rightarrow \infty} p_{2}^{n}=\lim _{n \rightarrow \infty} p_{1}^{n}=a_{1} \in Z
$$

Again, by taking subsequences, if necessary, we may assume that the sequence $\left\{a_{2}^{n}\right\}_{n=1}^{\infty}$ converges to some $a_{2} \in Z$. For concreteness, let us suppose $a_{2}-a_{1}>0$. Then, we can find $L>0$ and $N_{0} \in \mathbb{N}$, such that for all $n \geq N_{0}$ we have that

$$
F\left(a_{2}^{n}\right)-F\left(a_{1}^{n}\right) \geq L
$$

By Assumption 2.2, we have, that for every $y \in\left[a_{2}^{n}, a_{1}^{n}\right]$

$$
\mu\left(\left\{\alpha \in\left[a_{2}^{n}, a_{1}^{n}\right]: u\left(\varphi_{2}\left(p_{2}^{n}\right), \alpha\right) \geq u(y, \alpha)\right\}\right) \geq \delta\left(F\left(a_{2}^{n}\right)-F\left(a_{1}^{n}\right)\right)
$$

As in the proof of Lemma 5.3 this implies that

$$
F\left(\varphi_{2}\left(p_{2}^{n}\right)\right)-F\left(a_{1}^{n}\right) \geq \delta\left(F\left(a_{2}^{n}\right)-F\left(a_{1}^{n}\right)\right.
$$

and since, $\varphi_{2}\left(p^{n}\right) \leq p_{2}^{n}$,

$$
F\left(p_{2}^{n}\right)-F\left(a_{1}^{n}\right) \geq \delta\left(F\left(a_{2}^{n}\right)-F\left(a_{1}^{n}\right)\right) \geq \delta L>0
$$

for all $n \geq N_{0}$. But, this contradicts that $\lim _{n \rightarrow \infty} p_{2}^{n}=a_{1}$. A completely similar argument can be used to deal with the case $a_{2}=a_{1}$.

Proof of Theorem 6.1 We may proceed now as in section 5. That is we define the set

$$
\Sigma=\left\{\left(p_{1}, p_{2}, p_{3}\right) \in Z^{3}:\left|p_{i+1}-p_{i}\right| \geq \eta \text { for all } i=0, \ldots, 3\right\}
$$

(where $\eta>0$ is determined as in Lemma 6.2). Note that in the set $\Sigma$, we have that $\eta \leq p_{1} \leq 1-3 \eta, 2 \eta \leq p_{2} \leq 1-2 \eta, 3 \eta \leq p_{3} \leq 1-\eta$.

The truncated map $\bar{\varphi}: \Sigma \rightarrow \Sigma$ is defined successively as follows:

$$
\bar{\varphi}_{1}(p)= \begin{cases}\eta & \text { if } \varphi_{1}(p) \leq \eta \\ 1-3 \eta & \text { if } \varphi_{2}(p) \geq 1-3 \eta \\ \varphi_{1}(p) & \text { otherwise }\end{cases}
$$

That is, $\bar{\varphi}_{1}(p)=\min \left\{\max \left\{\varphi_{1}(p), \eta\right\}, 1-3 \eta\right\}$. It follows that $\bar{\varphi}_{1}$ is continuous. Define now,

$$
\bar{\varphi}_{2}(p)= \begin{cases}\bar{\varphi}_{1}(p)+\eta & \text { if } \varphi_{2}(p) \leq \bar{\varphi}_{1}(p)+\eta \\ 12 \eta & \text { if } \varphi_{2}(p) \geq 1-2 \eta \\ \varphi_{2}(p) & \text { otherwise }\end{cases}
$$


In other words, $\bar{\varphi}_{2}(p)=\min \left\{\max \left\{\bar{\varphi}_{1}(p)+\eta, \varphi_{2}(p)\right\}, 1-2 \eta\right\}$. And, since $\bar{\varphi}_{1}$ is continuous, so is $\bar{\varphi}_{2}$. Finally,

$$
\bar{\varphi}_{3}(p)= \begin{cases}\bar{\varphi}_{2}(p)+\eta & \text { if } \varphi_{3}(p) \leq \bar{\varphi}_{2}(p)+\eta \\ 1-\eta & \text { if } \varphi_{3}(p) \geq 1-\eta \\ \varphi_{3}(p) & \text { otherwise }\end{cases}
$$

And, since $\bar{\varphi}_{3}(p)=\min \left\{\max \left\{\bar{\varphi}_{2}(p)+\eta, \varphi_{3}(p)\right\}, 1-\eta\right\}$, we see that $\bar{\varphi}_{3}$ is also continuous.

Brouwer's Fixed Point Theorem applies again, so that there exists some $p^{*} \in \Sigma$ such that $\bar{\varphi}\left(p^{*}\right)=p^{*}$. Now, we must have that $\bar{\varphi}\left(p^{*}\right)=\varphi\left(p^{*}\right)=p^{*}$. For otherwise, let $i_{0}$ be the first party such that $\bar{\varphi}_{i_{0}+1}\left(p^{*}\right) \neq \varphi_{i_{0}+1}\left(p^{*}\right)$. That is, $\bar{\varphi}_{i_{0}}\left(p^{*}\right)=\varphi_{i_{0}}\left(p^{*}\right)=p_{i_{0}}^{*}$ for $i \leq i_{0}$. Then,

$$
\bar{\varphi}_{i_{0}+1}\left(p^{*}\right)-\bar{\varphi}_{i_{0}}\left(p^{*}\right)=p_{i_{0}+1}^{*}-p_{i_{0}}^{*}=\eta
$$

so, by Lemma 6.2 at least one of the two following inequalities is strict

$$
\varphi_{i_{0}}\left(p^{*}\right)<p_{i_{0}}^{*} \quad \text { or } \quad p_{i_{0}+1}^{*}<\varphi_{i_{0}+1}\left(p^{*}\right)
$$

But, in view that $\bar{\varphi}_{i_{0}}\left(p^{*}\right)=\varphi_{i_{0}}\left(p^{*}\right)=p_{i_{0}}^{*}$, we must have that $\varphi_{i_{0}+1}\left(p^{*}\right)-$ $\bar{\varphi}_{i_{0}}\left(p^{*}\right)>\eta$. Thus, $\bar{\varphi}_{i_{0}+1}\left(p^{*}\right)=\varphi_{i_{0}+1}\left(p^{*}\right)$, which contradicts the definition of $i_{0}$.

Remark 6.3 Of course, the above results only guarantees that in equilibrium there are three different proposals, but cannot exclude the case in which only two of them obtain a positive measure of votes. We do not have a general result to ensure existence of more than two positive-measure parties. However, the following generalized example shows that such equilibria are indeed easy to construct. In fact, for any population partition into intervals and for any set of party policy proposals we can find a population distribution such that these would correspond to an equilibrium! We also illustrate a striking feature of such equilibria: parties with tiny vote shares in equilibrium actually possess a significant political power. Of course, this underlines the role of subadditivity of policy outcome rules.

Example 6.4 (Generalized Example) Consider the case of three parties, with internal policy mappings $P_{i}(S)$ being the mean voting rule (the same statement is possible for the median voter rules). Let the function $g$ satisfy the condition in Equation 3.3 and $h(x)=x^{\gamma}, 0<\gamma<1$. Consider an arbitrary set of numbers $0<p_{1}<a_{1}<p_{2}<a_{2}<p_{3}<1$. There exists a population distribution that supports an equilibrium such that $P_{i}(S)=p_{i}, S_{1}=\left[0, a_{1}\right], S_{2}=\left[a_{1}, a_{2}\right], S_{3}=$ $\left[a_{2}, 1\right]$ and the policy outcome $T$ equals $a_{1}$ (or, if one wishes, $a_{2}$ ).

Before providing a proof for this claim, we shall make the following observations. Given any vector of party weights $\left(w_{1}, w_{2}, w_{3}\right) \in \Delta^{2}$ such that $w_{i}>0$ for 
$i=1,2,3$, it is always possible to find a continuous non-vanishing probability density function $f$ such that $\int_{0}^{a_{1}} f(t) \mathrm{d} t=w_{1}, \int_{a_{1}}^{a_{2}} f(t) \mathrm{d} t=w_{2}, \int_{a_{2}}^{1} f(t) \mathrm{d} t=w_{3}$. Furthermore, in addition we can always choose the density function so that the within-interval means match those in the example: $\frac{1}{w_{1}} \int_{0}^{a_{1}} t f(t) \mathrm{d} t=p_{1}$, $\frac{1}{w_{2}} \int_{a_{1}}^{a_{2}} t f(t) \mathrm{d} t=p_{2}, \frac{1}{w_{3}} \int_{a_{2}}^{1} t f(t) \mathrm{d} t=w_{3}$. Furthermore, the characterization conditions for the voting game SNE existence in Lemma 3.4 are independent of the within-party population distribution, but only depend on party weights $w_{i}$. Thus, if we find the positive population weights satisfying the conditions of the Lemma 3.4 for the chosen vector of party policies, the population partition and the policy outcome $T$, we shall demonstrate our claim.

To see that this is indeed always possible, consider that if the policy outcome is $T=a_{1}$ then by Lemma 3.4 the party weight vector $\left(w_{1}, w_{2}, w_{3}\right) \in \Delta^{2}$ must satisfy the following two conditions:

$$
\frac{a_{1}-p_{3}}{a_{1}-p_{2}}=\frac{h^{\prime}\left(w_{2}\right)}{h^{\prime}\left(w_{3}\right)}=\left(\frac{w_{3}}{w_{2}}\right)^{1-\gamma}
$$

and

$$
T(p, w)=\frac{w_{1}^{\gamma} p_{1}+w_{2}^{\gamma} p_{2}+w_{3}^{\gamma} p_{3}}{w_{1}^{\gamma}+w_{2}^{\gamma}+w_{3}^{\gamma}}=a_{1}
$$

Furthermore, since it has to be on a simplex (the entire population mass is split between parties)

$$
w_{1}+w_{2}+w_{3}=1
$$

Consider now 6.2. By multiplying both sides by the denominator and rearranging terms, we get

$$
w_{1}^{\gamma}\left(a_{1}-p_{1}\right)+w_{2}^{\gamma}\left(a_{1}-p_{2}\right)+w_{3}^{\gamma}\left(a_{1}-p_{3}\right)=0
$$

Dividing now both sides by $w_{2}^{\gamma}\left(a_{1}-p_{2}\right)$ we obtain

$$
\left(\frac{w_{1}}{w_{2}}\right)^{\gamma} \frac{\left(a_{1}-p_{1}\right)}{\left(a_{1}-p_{2}\right)}+1+\left(\frac{w_{3}}{w_{2}}\right)^{\gamma} \frac{\left(a_{1}-p_{3}\right)}{\left(a_{1}-p_{2}\right)}=0
$$

By substituting 6.1 into 6.5 we obtain

$$
\left(\frac{w_{1}}{w_{2}}\right)^{\gamma} \frac{\left(a_{1}-p_{1}\right)}{\left(a_{1}-p_{2}\right)}+1+\left(\frac{a_{1}-p_{3}}{a_{1}-p_{2}}\right)^{\frac{1}{1-\gamma}}=0
$$

We now can express the sufficient conditions for equilibrium in the voting game from Lemma 3.4 as a system of three linear (in party weights) equations in three 
variables:

$$
\begin{aligned}
w_{3}-\left(\frac{a_{1}-p_{3}}{a_{1}-p_{2}}\right)^{\frac{1}{1-\gamma}} w_{2} & =0 \\
w_{1}-\left(\left(1+\left(\frac{a_{1}-p_{3}}{a_{1}-p_{2}}\right)^{\frac{1}{1-\gamma}}\right) \frac{p_{2}-a_{1}}{a_{1}-p_{1}}\right)^{\frac{1}{\gamma}} w_{2} & =0 \\
w_{1}+w_{2}+w_{3} & =1
\end{aligned}
$$

It is clear that this system has a unique solution for every possible profile $0<p_{1}<a_{1}<p_{2}<a_{2}<p_{3}<1 .{ }^{5}$ Furthermore, such a solution must satisfy $w_{i}>0$ for $i=1,2,3$.

Given our observations in the beginning of this demonstration, we have proven our claim. The result for $T=a_{2}$ is symmetric.

To provide a numerical illustration for the above generalized example, consider that if $\gamma=\frac{1}{2}$ then $a_{1}=\frac{1}{3}, a_{2}=\frac{2}{3}, p_{1}=\frac{1}{6}, p_{2}=\frac{1}{2}, p_{3}=\frac{5}{6}, T=a_{2}=\frac{2}{3}$ characterizes a multi-party pluralistic equilibrium with three positive-mass parties for any population distribution such that $\int_{0}^{\frac{1}{3}} f(t) \mathrm{d} t=\frac{9}{110}, \int_{\frac{1}{3}}^{\frac{2}{3}} f(t) \mathrm{d} t=\frac{1}{110}$, $\int_{\frac{2}{3}}^{1} f(t) \mathrm{d} t=\frac{100}{110}$ and $\frac{110}{9} \int_{0}^{\frac{1}{3}} t f(t) \mathrm{d} t=\frac{1}{6}, 110 \int_{\frac{1}{3}}^{\frac{2}{3}} t f(t) \mathrm{d} t=\frac{1}{2}$, and $\frac{110}{100} \int_{\frac{2}{3}}^{1} t f(t)$ $\mathrm{d} t=\frac{5}{6}$ (that this does indeed constitute an equilibrium is easy to check directly, since any shift of population mass from party 3 makes the policy outcome move to the left of $\frac{2}{3}$ and any shift of population mass between or out of the parties 1 and 2 makes the policy outcome shift to the right of $\frac{2}{3}$ ). In this example, the leftist and, especially, the centrist parties get only a small proportion of voters, but have a far from negligible impact on the policy outcome.

To end this section, we provide an example of non-existence of pluralistic multiparty equilibrium in which all three parties receive a positive vote share for a given voter distribution. In particular, if the population distribution is uniform, equilibria of this type do not exist.

Example 6.5 Suppose that the distribution of agents is uniform on the interval $Z=[0,1]$. There are three parties and the mapping $P$ satisfies that $p_{i}=P_{i}(S)$ is the median type of $S_{i}, i=1,2,3$. The function $g$ satisfies condition in Eq. 3.3 and $h(x)=x^{\gamma}, 0<\gamma<1$.

Since $S_{i}$ is an interval in the real line the median type is well defined and unique. Let $w_{i}$ be the mass of interval $S_{i}, i=1,2,3$. Suppose that there exists a multiparty equilibrium under which the three parties receive a positive vote

\footnotetext{
5 Since both $\left(\frac{a_{1}-p_{3}}{a_{1}-p_{2}}\right)^{\frac{1}{1-\gamma}}>1$ and $\left(\left(1+\left(\frac{a_{1}-p_{3}}{a_{1}-p_{2}}\right)^{\frac{1}{1-\gamma}}\right) \frac{p_{2}-a_{1}}{a_{1}-p_{1}}\right)^{\frac{1}{\gamma}}>0$, the singularity condition for the left hand side $\left(\frac{a_{1}-p_{3}}{a_{1}-p_{2}}\right)^{\frac{1}{1-\gamma}}+\left(\left(1+\left(\frac{a_{1}-p_{3}}{a_{1}-p_{2}}\right)^{\frac{1}{1-\gamma}}\right) \frac{p_{2}-a_{1}}{a_{1}-p_{1}}\right)^{\frac{1}{\gamma}}=1$ cannot be satisfied.
} 
share, i.e. $0<w_{i}<1, i=1,2,3$. Then, it must be true that

$$
p_{1}=\frac{w_{1}}{2}, p_{2}=w_{1}+\frac{w_{2}}{2}, p_{3}=w_{1}+w_{2}+\frac{w_{3}}{2}
$$

Furthermore, the necessary conditions in Lemma 3.4 have to be satisfied. Say that, without loss of generality, the first condition in the Lemma is satisfied, Thus, we have

$$
T(p, w)=w_{1}+w_{2}
$$

In this case, Condition (3.1) of the same Lemma requires that

$$
\frac{T(p, w)-p_{1}}{T(p, w)-p_{2}}=\frac{h^{\prime}\left(w_{2}\right)}{h^{\prime}\left(w_{1}\right)}
$$

Conditions 6.8-6.10 imply

$$
\frac{w_{1}+2 w_{2}}{w_{2}}=\frac{w_{2}^{\gamma-1}}{w_{1}^{\gamma-1}}
$$

that can be written as

$$
w_{1}=w_{1}^{1-\gamma} w_{2}^{\gamma}-2 w_{2}
$$

We have already seen that concavity of $h$ and inequality 6.10 imply that $w_{1}>w_{2}$. Hence,

$$
w_{1}^{1-\gamma} w_{2}^{\gamma}-2 w_{2}<w_{1}-2 w_{2}
$$

but inequality in 6.12 contradicts equality 6.10 .

Acknowledgements The manuscript has benefited substantially thanks to the careful reading of an anonymous referee. We are greatly indebted for his/her generous comments, and, especially, for indicating an important error in an earlier draft. Ortuño-Ortín is grateful for the financial support of the Comunidad de Madrid (grant 06/HSE/0157/200) and of the Fundacion BBVA. Marhuenda is grateful for the financial support from the Spainsh Ministry of Education (grant SEJ2004-00968). Gomberg is grateful to the Asociación Mexicana de Cultura for financial support.

\section{References}

Aldrich JH (1983) A Downsian spatial model of party activism. Am Polit Sci Rev 77:974-990 Alesina A, Rosenthal H (1997) A theory of divided government. Econometrica 64:1311-134 Aumann RJ (1959) Acceptable points in general cooperative $n$-person games. In: Tucker AW,

Luce RD (eds) Contributions to the theory of games. vol IV (Annals of Mathematics Studies, 40) Princeton University Press, Princeton, pp 287-324

Baron D (1993) Government formation and endogenous parties. Am Polit Sci Rev 87:34-47 
Besley T, Coate S (1997) An economic model of representative democracy. Quart J Econ (February) 85-114

Caplin A, Nalebuff B (1997) Competition among institutions. J Econ Theory 72:306-342

Gerber A, Ortuño-Ortín I (1998) Political compromise and endogenous formation of coalitions. Soc Choice Welf 15:445-454

Gomberg A (2004) Sorting equilibrium in a multi-jurisdiction model. J Econ Theory 116:138-154

Gomberg A (2005) An example of non-existence of three-community equilibrium. J Publ Econ Theory 7:285-294

Gomberg A, Marhuenda F, Ortuño-Ortín I (2004) A model of endogenous political party platforms. Econ Theory 24:373-394

Levy G (2004) A model of political parties. J Econ Theory 115(2):250-277

Ortuño-Ortín I (1997) A spatial model of political competition and proportional representation. Soc Choice Welf 14:427-438

Ortuño-Ortín I, Roemer JE (1998) Endogenous party formation and the effect of income distribution on policy. Mimeo

Osborne MJ, Al Slivinski (1996) A model of political competition with citizen-candidates. Quart J Econ CXI:65-96

Osborne MJ, Tourky R (2004) Party formation in single-issue politics. Manuscript

Poutvaara P (2003) Party platforms with endogenous party membership. Publ Choice 117:77-98

Roemer JE (1999) The democratic political economy of progressive taxation. Econometrica 67:1-20

Westhoff F (1977) Existence of equilibrium with a local public good. J Econ Theory 14:84-112 